\title{
Delineation of mechanisms and regions of dosage imbalance in complex rearrangements of 1 p36 leads to a putative gene for regulation of cranial suture closure
}

\author{
Marzena Gajecka ${ }^{1}$, Wei $\mathrm{Yu}^{3}$, Blake C Ballif ${ }^{1,3}$, Caron D Glotzbach ${ }^{1,2}$, Kristen A Bailey ${ }^{1,2}$, \\ Chad A Shaw ${ }^{3}$, Catherine D Kashork ${ }^{3}$, Heidi A Heilstedt ${ }^{3}$, David A Ansel ${ }^{4}$, Aaron Theisen ${ }^{1}$, \\ Ritva Rice ${ }^{5}$, David PC Rice ${ }^{5}$ and Lisa G Shaffer ${ }^{*, 1,2}$

\begin{abstract}
${ }^{1}$ Health Research and Education Center, Washington State University, Spokane, WA, USA; ${ }^{2}$ Sacred Heart Medical Center, Spokane, WA, USA; ${ }^{3}$ Department of Molecular and Human Genetics, Baylor College of Medicine, Houston, TX, USA; ${ }^{4}$ Department of Medicine, Children's Hospital, Boston, MA, USA; Department of Craniofacial Development, King's College, London
\end{abstract}

Structural chromosome abnormalities have aided in gene identification for over three decades. Delineation of the deletion sizes and rearrangements allows for phenotype/genotype correlations and ultimately assists in gene identification. In this report, we have delineated the precise rearrangements in four subjects with deletions, duplications, and/or triplications of $1 \mathrm{p} 36$ and compared the regions of imbalance to two cases recently published. Fluorescence in situ hybridization (FISH) analysis revealed the size, order, and orientation of the duplicated/triplicated segments in each subject. We propose a premeiotic model for the formation of these complex rearrangements in the four newly ascertained subjects, whereby a deleted chromosome 1 undergoes a combination of multiple breakage-fusion-bridge (BFB) cycles and inversions to produce a chromosome arm with a complex rearrangement of deleted, duplicated and triplicated segments. In addition, comparing the six subjects' rearrangements revealed a region of overlap that when triplicated is associated with craniosynostosis and when deleted is associated with large, late-closing anterior fontanels. Within this region are the MMP23A and - $B$ genes. We show MMP23 gene expression at the cranial sutures and we propose that haploinsufficiency results in large, late-closing anterior fontanels and overexpression results in craniosynostosis. These data emphasize the important role of cytogenetics in investigating and uncovering the etiologies of human genetic disease, particularly cytogenetic imbalances that reveal potentially dosage-sensitive genes.

European Journal of Human Genetics (2005) 13, 139-149. doi:10.1038/sj.ejhg.5201302

Published online 13 October 2004

Keywords: complex rearrangements; 1p36; cranial suture closure; MMP23

Introduction

The identification of the chromosomal and genetic basis of multiple malformation syndromes has primarily relied

*Correspondence: Dr LG Shaffer, Health Research and Education Center, Washington State University, Box 1495, Spokane, WA 99210-1495, USA. Tel: 509368 6710; Fax: 509358 7627; E-mail: Ishaffer@wsu.edu Received 13 April 2004; revised 21 July 2004; accepted 1 September 2004 upon the use of positional cloning, breakpoint analysis, and other labor-intensive strategies to identify the genes responsible for particular clinical phenotypes. These strategies require complete genetic maps of the region, the availability of large-insert clones, patients harboring translocations or other chromosomal anomalies, and correlations between phenotype and genotype. One of the first instances in which structural chromosomal 
abnormalities were used in gene identification was the localization of the genes for cytoplasmic isocitrate dehydrogenase (IDH-1) and cytoplasmic malate dehydrogenase (MDH-1) on chromosome 2 using cells with a balanced reciprocal translocation $\mathrm{t}(1 ; 2)(\mathrm{q} 32 ; \mathrm{q} 13) .{ }^{1}$ In a more recent example, the elastin gene was identified to be deleted in the majority of individuals with Williams syndrome $^{2}$ after a family with isolated supravalvular aortic stenosis (SVAS), a commonly occurring feature in Williams syndrome, was found to have a translocation involving chromosome 7 that disrupted the elastin gene. ${ }^{3}$ Other examples exist of structural chromosome abnormalities aiding in the identification of genes involved in microdeletion syndromes (eg, Rubinstein-Taybi syndrome $^{4}$ ). Finally, patients with overlapping deletions of varying sizes and phenotypes have aided in construction of natural deletion panels and the ordering of putative genes within a chromosomal region and ultimately gene identification (eg, Xp22.3 contiguous gene syndrome ${ }^{5}$ ). For those deletion syndromes that result from recombination through flanking low-copy repeat sequences, resulting in deletions of a consistent size, ${ }^{6}$ gene identification is particularly difficult because most patients have most features of the syndrome and all patients have the samesized deletions with the same breakpoints. ${ }^{7}$ Ideally, those syndromes with overlapping deletion regions and common clinical features should be well-suited to identify the causative genes.

Monosomy $1 \mathrm{p} 36$ is a contiguous gene syndrome resulting from a heterozygous terminal deletion of the most distal chromosomal band on the short arm of chromosome 1. Occurring in $\sim 1$ in 5000 births, monosomy 1p36 is the most commonly observed terminal deletion. ${ }^{8,9}$ Monosomy 1 p36 is associated with mental retardation, hearing impairment, seizures, growth impairment, and heart defects. In addition, the syndrome is characterized by several distinct craniofacial features, including late-closing anterior fontanels, brachycephaly, pointed chin, flat nasal bridge, and deep-set eyes. ${ }^{10-12}$ Because the deletion sizes in $1 \mathrm{p} 36$ are variable, ranging from $\sim 1.5$ to $>10.5 \mathrm{Mb}^{12,13}$ and, for some features, the occurrence corresponds with the sizes of the deletions, a genotype/ phenotype correlation should be more informative in monosomy 1p36 than for the many interstitial deletion syndromes that have common-sized deletions. Several candidate genes for features of 1 p36 deletion syndrome, including facial clefting anomalies $\left(S K I^{14}\right)$ and seizures $\left(K C N A B 2^{15}\right)$ have been proposed following genotype/ phenotype comparison.

Although deletions of $1 \mathrm{p}$ are relatively common among chromosome abnormalities, duplications and triplications of the short arm of chromosome 1 are rare. In all, 14 cases with interstitial duplications of $1 p$ have been reported. ${ }^{16-18}$ Although several of the common clinical manifestations have been reviewed, ${ }^{17}$ a consensus duplication syndrome has not been clinically defined due to the variety of breakpoints and lack of common segments involved.

A gene responsible for the large, late-closing anterior fontanels in some monosomy 1 p36 patients has not been proposed. Herein, we propose a candidate gene based on the analysis of several subjects with deletions of $1 p$ presenting with large, late-closing anterior fontanels and two subjects with duplications and triplications of $1 \mathrm{p}$ with craniosynostosis.

\section{Subjects and methods Subjects}

Over 90 subjects with rearrangements of the most distal portion of 1 p36 have been ascertained for investigation of deletion sizes, characterization of rearrangements, and phenotype/genotype correlations; 61 of these were recently published. ${ }^{12}$ Six subjects were identified to have deletions, duplications, and/or triplications of 1p36. Subjects 30, 40 and 69 have been described previously (subjects 30 and $40^{19}$; subject $69^{16}$ ). Subjects 30 and 40 presented with large, late-closing anterior fontanels. By physician or parental report, subjects 64 and 85 did not have craniosynostosis, although time of fontanel closure was not noted or revealed to be remarkable. However, subjects 69 and 71 presented with craniosynostosis. Subject $69^{16}$ displayed metopic synostosis, whereas subject 71 presented with sagittal and partial coronal synostosis. In addition, subjects 69 and 71 had microcephaly, developmental delay, structural heart defects (ASD (subject 69) or ASD/PDA (subject 71)), and eye anomalies (blepharophimosis (subject 69) or ptosis (subject 71)). Additional clinical features of subject 69 were published ${ }^{16}$ and are compared to subject 71 in Table 1 .

\section{Array CGH analysis}

Subject DNA was used in array CGH analyses. The construction of the microarray ${ }^{20}$ and BAC/PAC clones used $^{12}$ have been published. Genomic DNA was isolated from lymphoblastoid cell lines established from four subjects $(64,69,71,85)$ with known 1 p36 rearrangements and from peripheral blood of a phenotypically normal male reference. Microarray analysis for subjects 30 and 40 was reported previously. ${ }^{19}$ We used a dye-reversal strategy on two separate microarrays in which study subject and reference DNAs were labeled with cyanine 3 (Cy3) and cyanine 5 (Cy5), respectively, cohybridized to one microarray and then oppositely labeled and cohybridized to a second microarray. ${ }^{20,21}$ Images were acquired using a GenePix 4000B (Axon Instruments) dual-laser scanner in combination with GenePix Pro 4.0 imaging software. Two simultaneous scans of each array were obtained at wavelengths of 635 and $532 \mathrm{~nm}$, and data analysis was performed as described previously ${ }^{20}$ to identify gains or losses within this particular region of 1 p36. 
Table 1 Comparison of clinical features in subjects 69 and 71

\begin{tabular}{|c|c|c|}
\hline & Subject 69 & Subject 71 \\
\hline \multirow[t]{6}{*}{ Craniofacial } & Bitemporal narrowing & Low-set ears \\
\hline & Sloping forehead & Upturned nose \\
\hline & & Mild hypertelorism \\
\hline & Metopic synostosis & Sagittal and partial coronal synostosis \\
\hline & Microcephaly & Microcephaly \\
\hline & & Mild dilation of lateral ventricles \\
\hline \multirow[t]{3}{*}{ Ocular } & Blepharophimosis & Bilateral ptosis \\
\hline & & Intermittent exotropia \\
\hline & & hypermetropia \\
\hline Cardiovascular & ASD & PDA, ASD \\
\hline \multirow[t]{2}{*}{ Trunk } & Rectal stenosis & Anterior placement of anus \\
\hline & & Sacral dimple with tethered cord \\
\hline \multirow{3}{*}{ Central nervous system } & Developmental delay & Severe global developmental delays \\
\hline & & Hypotonia at birth \\
\hline & & Swallowing dysfunction \\
\hline
\end{tabular}

\section{FISH analyses}

FISH was performed on metaphase chromosomes on all subjects using the Cytocell Multiprobe ${ }^{\mathrm{T}}$ (Cambridge, UK) subtelomere FISH probe set as previously described. ${ }^{22}$ Additional FISH analysis for each subject was performed in three steps: metaphase FISH to confirm the deletions and interphase FISH to confirm the duplications and triplications; interphase FISH to establish the order of the rearranged segments relative to the centromere; and interphase FISH to determine the orientation of the segments relative to each other. The BAC clones used to confirm the deletions, duplications, and triplications and ascertain the order and orientation in each of the six subjects are shown in Table 2 . Because probes to duplicated and triplicated regions should display multiple signals (one and two extra signals, respectively), we used a three-color FISH analysis to determine the order of the rearranged segments relative to each other in which differentially labeled probes were assigned to each copy-number change: biotin-FITC (green) was assigned to triplications, digoxigenin-rhodamine (red) was assigned to duplications, and yellow (mixture of biotin and digoxigenin labels) was assigned to normal segments. A similar process was used to determine the orientation of each rearranged segment to the centromere. For each rearranged segment, two different-colored BAC probes were used: one proximal (centromeric), one distal (telomeric). To simplify the analysis, we performed FISH separately for duplications and triplications when determining the orientations (see Figure 1 legend for details).

DNA was extracted from BAC clones using a standard alkaline lysis protocol and labeled by nick translation (Roche Diagnostic, Indianapolis, IN, USA) with biotindUTP or digoxigenin-dUTP (Roche) or both. Two-color FISH was performed as previously described. ${ }^{23}$ The slides were counterstained with DAPI and digital images were
Table 2 BAC clones used in FISH analysis to confirm the deletion, duplication, and triplication sizes and determine the order and orientation of the rearranged segments

\begin{tabular}{|c|c|c|c|c|}
\hline Subject & Deletion & Duplication & Triplication & Normal \\
\hline 64 & $\begin{array}{l}\text { RP4-740C4 } \\
\text { RP11-361M21 } \\
\text { RP4-713A8 } \\
\text { RP4-758J18 }\end{array}$ & $\begin{array}{l}\text { RP3-395M20 } \\
\text { RP4-755G5 } \\
\text { RP13-436F16 }\end{array}$ & No trp & $\begin{array}{l}\text { RP1-163G9 } \\
\text { RP11-168F9 }\end{array}$ \\
\hline 69 & RP5-857K21 & $\begin{array}{l}\text { RP11-181G12 } \\
\text { RP4-713A8 } \\
\text { RP1-163G9 } \\
\text { RP4-785P20 } \\
\text { RP11-168F9 } \\
\text { RP11-495K24 } \\
\text { RP5-1166F10 } \\
\text { RP5-1098C18 }\end{array}$ & $\begin{array}{l}\text { RP11-54O7 } \\
\text { RP5-890O3 } \\
\text { RP4-758J18 } \\
\text { RP1-283E3 }\end{array}$ & $\begin{array}{l}\text { RP1-120G22 } \\
\text { RP1-202O8 } \\
\text { RP11-58A11 } \\
\text { RP1-126A5 } \\
\text { RP5-1113E3 }\end{array}$ \\
\hline 71 & $\begin{array}{l}\text { RP5-857K21 } \\
\text { RP11-54O7 } \\
\text { RP11-465B22 } \\
\text { RP5-902P8 }\end{array}$ & $\begin{array}{l}\text { RP11-168B8 } \\
\text { RP5-1166F10 } \\
\text { RP1-37J18 } \\
\text { RP11-319A11 }\end{array}$ & $\begin{array}{l}\text { RP5-89003 } \\
\text { RP4-758J18 } \\
\text { RP1-283E3 } \\
\text { RP4-713A8 } \\
\text { RP1-163G9 } \\
\text { RP4-785P20 } \\
\text { RP11-168F9 } \\
\text { RP11-495K24 } \\
\text { RP11-374C13 }\end{array}$ & $\begin{array}{l}\text { RP11-493P12 } \\
\text { RP5-1096P7 } \\
\text { RP11-58A11 } \\
\text { RP1-126A5 } \\
\text { RP5-1113E3 }\end{array}$ \\
\hline 85 & $\begin{array}{l}\text { RP4-785P20 } \\
\text { RP13-436F16 }\end{array}$ & $\begin{array}{l}\text { RP5-1092A11 } \\
\text { RP1-286D6 }\end{array}$ & No trp & $\begin{array}{l}\text { RP11-168F9 } \\
\text { RP11-495K24 } \\
\text { RP11-374C13 }\end{array}$ \\
\hline
\end{tabular}

captured using an Applied Imaging system (Carlsbad, CA, USA).

\section{Microsatellite marker analysis}

To determine the parental origin of the deleted regions in subjects 64, 69, 71, and 85, microsatellite marker analysis was performed on PCR-amplified genomic DNA from the subjects and their available parents. Genotyping was performed using an ABI PRISM 3100-Avant genetic analyzer (Applied Biosystems). The DS-30(D) dye set was used 

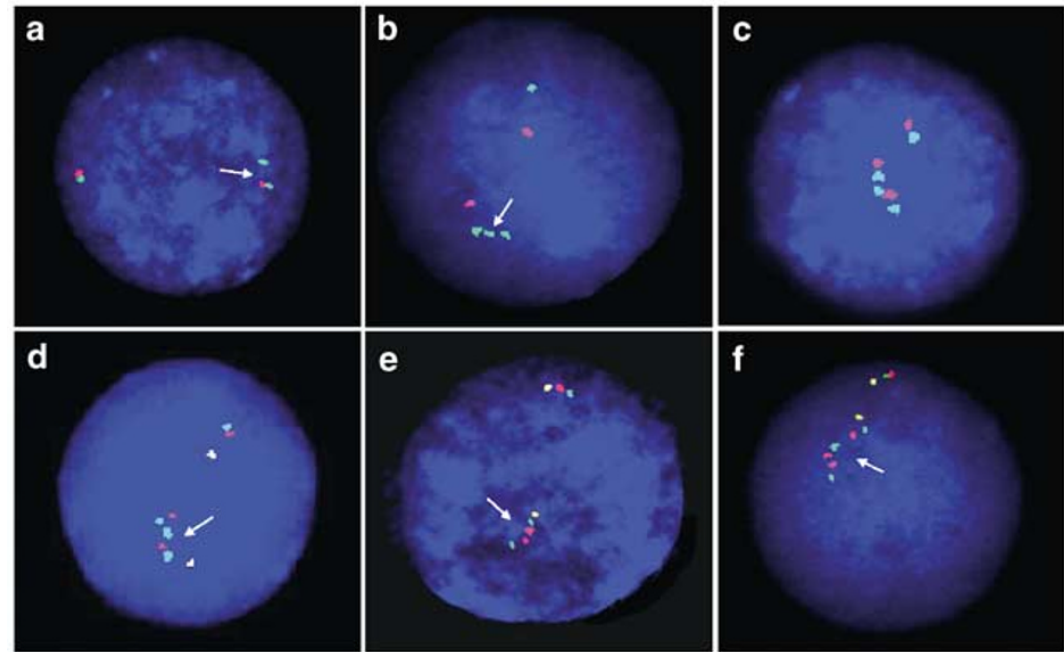

Figure 1 Representative interphase FISH results for subject 69. (a) BAC RP5-1098C18 from the duplicated segment is labeled in green and BAC RP1-120G22, labeled in red, is from a nonrearranged segment. The green signal pattern is consistent with a duplication (arrow). (b) BAC RP1-283E3 from the triplicated segment is labeled in green and BAC RP11-58A11, labeled in red, is from a nonrearranged segment. The green signal pattern is consistent with a triplication (arrow). (c) BAC RP11-181G12 from the duplicated segment (red) and BAC RP1-283E3 from the triplicated segment (green) shows the orientation of each segment relative to one another. (d) The same experiment as in (c), with the addition of an 'anchor' from the nonrearranged, proximal (centromeric) segment (BAC RP1-120G22, yellow signal) reveals the orientation of the segments relative to each other and the centromere (arrow). (e) The orientation of the duplicated segment is revealed using BAC RP11-181G12, labeled in green, and BAC RP1-163G9 labeled in red. A proximal 'anchor' (BAC RP11-58A11, yellow) is used to determine that the duplicated segment is in an inverted orientation (yellow, green, red, red, green; arrow). (f) The orientation of the triplicated segment is revealed using BAC RP1-283E3, labeled in green, and BAC RP4-758)18 labeled in red. A proximal 'anchor' (BAC RP1-120G22, yellow) is used to determine that the triplicated segments are in tandem for two copies, while one copy is inverted (yellow, green, red, green, red, red, green; arrow).

according to the manufacturer's specifications. Primers were obtained labeled with 5'-HEX or 5'6-FAM (Integrated DNA Technologies, Inc.). The ABI PRISM GeneScan analysis software and ABI PRISM Genotyper software were used to analyze the results.

\section{Gene identification}

The gene content of the $1.1 \mathrm{Mb}$ region between $\mathrm{BAC}$ clones RP5-890O3 and RP11-547D24 was identified by comparative analysis of the annotated electronic databases of three public genome browsers: the NCBI genome browser (http://www.ncbi.nlm.org), the UCSC genome browser (http://www.genome.ucsc.edu), and Ensembl (http:// www.ensembl.org). Because the number of hypothetical/ predicted genes varies between databases, only the known genes and novel genes with functional identifiers found in at least two of the three databases were included in Table 3.

\section{In situ hybridization analyses}

An Mmp23 mRNA probe was prepared from a $308 \mathrm{bp}$ fragment of rat Mmp23 cDNA in Bluescript II SK+ (Stratagene) (a gift from J Ohnishi) by digesting with EcoRI. In situ hybridization was performed according to
Vainio et $a l^{49}$ using ${ }^{35} \mathrm{~S}$ UTP-labeled riboprobes on tissue sections of coronal and sagittal sutures dissected from embryonic CD1 mice. Both bright- and dark-field images were taken of hybridized sections. Silver grains were selected from the dark-field image, colored red, and superimposed onto the identical bright-field image.

\section{Results \\ Characterization of rearrangements of 1 p36}

Array CGH revealed DNA copy number changes in all four subjects. In subject 69 , copy-number gains indicated an $\sim 1.25 \mathrm{Mb}$ terminal triplication followed by an $\sim 4 \mathrm{Mb}$ duplication (Figures $2 \mathrm{a}$ and 3). Microarray analysis for subject 71 revealed a $1 \mathrm{Mb}$ terminal deletion, followed by an $\sim 2.8 \mathrm{Mb}$ triplication and a more proximal duplication spanning $\sim 400 \mathrm{~kb}$ (Figures $2 \mathrm{~b}$ and 3 ). In subject 64 , array $\mathrm{CGH}$ revealed a $2 \mathrm{Mb}$ deletion followed by a $\sim 0.4 \mathrm{Mb}$ duplication (Figure 3). In subject 85, array CGH indicated a deletion of $\sim 3 \mathrm{Mb}$ followed by a $\sim 350 \mathrm{~kb}$ duplication (Figure 3).

To delineate the order of the rearranged segments and determine their orientation with respect to the centromere, 
Table 3 Known genes in 1p36.3 located in the critical region for suture closure regulation between RP5-890O3 and RP11547D24

Gene symbol Full gene name

MRPL20 Mitochondrial ribosomal protein L20

WARP von Willebrand factor A domain-related protein

TOB3 AAA-ATPase TOB3

MMP23A/B Matrix metalloproteinase 23A/B

CDC2L1/2 Cell division cycle 2-like 1/2 (PITSLRE protein) (p58)

SLC35E2 Solute carrier family 35, member E2

PPNK NAD kinase

GNB1 Guanine nucleotide binding protein (G protein), beta polypeptide 1

$G A B R D$

Gamma-aminobutyric acid (GABA) A receptor, delta

PRKCZ Protein kinase C, zeta

V-ski sarcoma viral oncogene homolog (avian)

RER1 RER1 homolog (Saccharomyces cerevisiae)

PEX10 Peroxisome biogenesis factor 10

PANK4 Pantothenate kinase 4

HES5 Hairy and enhancer of Split, Drosophila, homolog of 5; bHLH factor Hes5

TNFRSF14 Tumor necrosis factor receptor superfamily, member 14 (herpesvirus entry mediator)
Known function

Protein synthesis within the mitochondrion. ${ }^{24}$

Expressed predominantly in chondrocytes and suggested to be a novel member of a superfamily of extracellular matrix proteins which may play a role in cartilage structure and function. ${ }^{25,26}$

Suggested to be a novel member of the AAA-ATPase family which often performs chaperone-like functions in a variety of cellular processes. A number of AAA-ATPase family members are responsible for various human diseases (eg, peroxisomal disorders, paraplegias, lissencephaly). ${ }^{27}$ Suggested to be a novel member of the TOB family of antiproliferative proteins. TOB proteins have recently been shown to play an important role in osteogenesis by inhibiting bone morphogenetic protein (BMP) signaling through interacting with

Smad-receptors in osteoblasts. ${ }^{28,29}$

MMP family members are involved in the breakdown of extracellular matrix in normal physiological processes, such as embryonic development, reproduction, and tissue remodeling, as well as in disease processes, such as arthritis and metastasis. ${ }^{30}$ Possible role in endochondral bone formation. ${ }^{31}$

Protein kinase cleaved by caspases and demonstrated to play a role in apoptosis. ${ }^{32}$ Negative regulator of normal cell cycle progression. Suggested to be involved in a variety of cancers. ${ }^{33}$

Novel member of the solute carrier family that encodes nucleotide sugar transporters localized to the Golgi and/or the ER. In model systems these transporters are involved in tumor metastasis, cellular immunity, organogenesis, and morphogenesis. Other roles in connective tissues and muscles have been suggested. ${ }^{34}$

Putative NAD kinase involved in the synthesis of NADP which is essential for biosynthetic pathways, energy, and signal transduction ${ }^{35}$ Regulates alpha subunits of $G$ proteins. Heterotrimeric guanine nucleotide-binding proteins ( $G$ proteins) transduce extracellular signals received by transmembrane receptors to effector proteins. The beta1gamma1 dimer is the primary dimer in retina and beta1gamma2 is the primary dimer in the brain. Erythrocyte membranes contain beta subunit types $1,2,3$, and $4 .^{36,37}$

Encodes one of at least 15 ligand-gated chloride channels for gammaaminobutyric acid (GABA), the major inhibitory neurotransmitter in the mammalian brain. 'Tonic' or continuous conductance in neurons is lost when gene is knocked out in mice, leading to a change in normal neuronal behavior. ${ }^{38}$

Member of the PKC family of serine/threonine kinases which are involved in a variety of cellular processes such as proliferation, differentiation, and secretion. Plays an important role in insulinstimulated glucose transport and may be associated with type II diabetes. Knockout mice show that PKCZ plays a role in the NFKappaB pathway. ${ }^{39}$

Component of the TGFB1 signaling pathway. Involved in neural tube development and muscle differentiation. ${ }^{40}$ Depending on the genetic background, SKI-/- mice show midline facial clefting and other defects. ${ }^{14}$

Golgi membrane protein required for the correct localization of ER membrane proteins by a retrieval mechanism from the cis-Golgi to the $\mathrm{ER}^{41}$

Involved in import of peroxisomal matrix proteins. PEX10 mutations result in a range of peroxisomal biogenesis disorders from neonatal adrenoleukodystrophy to Zellweger syndrome ${ }^{42}$ that are characterized by neuronal, hepatic, and renal abnormalities and severe mental retardation.

Key regulatory enzyme in the biosynthesis of coenzyme $A .{ }^{43}$

Hes family members play various roles in the Notch signaling pathway which is involved in neurogenesis and organ development. ${ }^{44,45}$ Hes5 may play an important role in hair development in the cochlea. ${ }^{46}$ Members of the TNFR family play a key role in regulating the immune response to infection. Isolated as a herpesvirus entry mediator protein. ${ }^{47}$ Activates JNK1, NF-KappaB, and AP1 which control expression of multiple genes in response to infection or cellular stress. $^{48}$ 

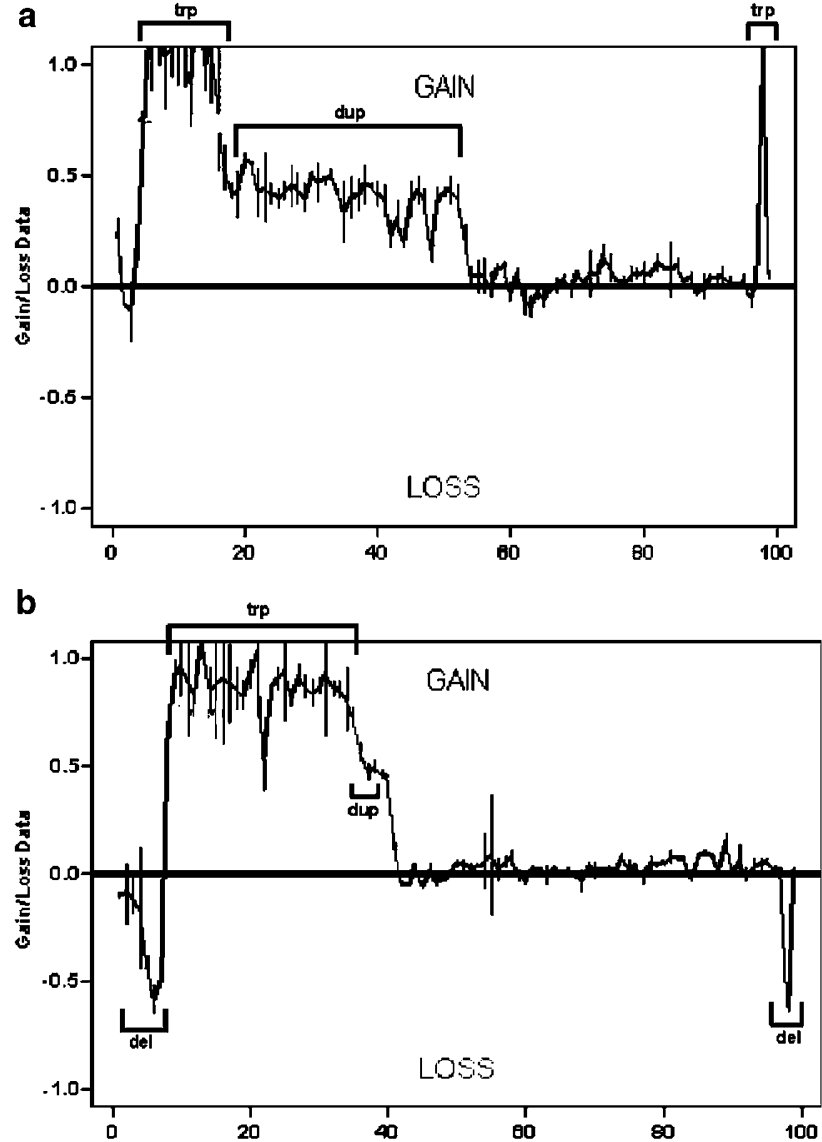

Figure 2 Representative plots of the array $\mathrm{CGH}$ hybridization results for (a) subject 69 and (b) subject 71. Each shows the individual signal ratios (scale on left) for the 97 BAC clones from the distal 1 p36 10.5 Mb contig ${ }^{12}$ used on the microarray. A ratio above $0.65\left(s^{2}= \pm 0.073\right)$ indicates copy-number gain, a ratio below $-0 . \overline{567}\left(s^{2}= \pm 0.02\right)$ indicates copy-number loss, and a ratio of 0.005 $\left(s^{2}= \pm 0.009\right)$ indicates a normal copy number. ${ }^{20}$ In subject 69 (a), array $\mathrm{CGH}$ indicated a $\sim 1.25 \mathrm{Mb}$ terminal triplication (trp) followed by a $\sim 4 \mathrm{Mb}$ duplication (dup). In subject 71 (b), array $\mathrm{CGH}$ revealed $\mathrm{a} \sim 1 \mathrm{Mb}$ terminal deletion (del), a $\sim 2.8 \mathrm{Mb}$ triplication (trp), and a more proximal $400 \mathrm{~kb}$ duplication (dup).

we performed a multistep FISH analysis to reconstruct the rearranged chromosomes 1 . In subject 69, FISH revealed that RP5-857K21, the most distal clone on the $1 \mathrm{p} 36$ contig, was deleted. Because this clone resides in the telomereassociated repeat (TAR) region and is shared by multiple nonhomologous chromosome ends, ${ }^{12}$ FISH was performed on the subject's parents to clarify whether this was truly a deletion or a polymorphism. FISH on each parental sample showed hybridization to both chromosomes 1 , indicating that the deletion of a single clone in subject 69 is de novo and not a polymorphic variant (data not shown). Array
CGH was unable to detect this copy-number loss due to its location in multiple TAR regions. ${ }^{20}$ FISH in subjects 64,71 and 85 confirmed their deletions. Likewise, single FISH experiments confirmed the regions of duplication and/or triplication in all subjects.

In all subjects, subtelomere FISH was performed. The $1 \mathrm{p}$ subtelomere region was deleted in all subjects except subject 69 . Additionally, only subject 71 showed hybridization of the rearranged chromosome 1 with another subtelomere clone. FISH demonstrated three copies of $19 q$, one on each chromosome 19 and one on the distal short arm of the derivative 1 (data not shown).

We next performed interphase FISH to determine the order of the rearranged segments to each other in each subject. Determining the order of the rearranged segments is necessary for reconstructing the rearranged chromosomes. Representative FISH results for subject 69, shown in Figure 1, demonstrate the use of FISH to reconstruct the rearrangements.

Using the FISH results, we derived the minimum steps required to construct the rearranged chromosomes from the normal chromosome 1 . Shown in Figure 4 is the reconstruction of the rearrangement for subject 71 . Reconstruction of the rearrangements is necessary for understanding the mechanisms of formation.

Parental origin analysis was performed on subjects 64 , 69,71 , and 85 . Subjects 64 and 85 were shown to have deletions of paternal origin. Because of the small deletion sizes in subjects 69 and 71, no informative microsatellite markers were available. The deletions in subjects 30 and 40 were maternally derived. ${ }^{19}$

\section{Identification of MMP23 as a candidate gene for cranial suture closure}

Because two subjects with deletion, duplication and triplication had craniosynostosis and four subjects with deletion and duplication had large, late-closing anterior fontanels characteristic of monosomy $1 \mathrm{p} 36$, we sought to identify a gene on 1 p36 that plays a role in regulating cranial suture closure. To accomplish this, we compared the regions of deletion, duplication and triplication among the six subjects (Figure 3), with the hypothesis that those individuals with large, late-closing anterior fontanels will be deleted for the region containing the gene and those individuals with craniosynostosis will be duplicated or triplicated for the region containing the gene. This comparison revealed a $1.1 \mathrm{Mb}$ region of overlap that is likely to contain a gene that plays a role in regulating cranial suture closure. This region is defined by RP5-890O3 distally and RP11-547D24 proximally (Figure 3). Within this region, 18 known genes reside (Table 3 ). We selected the matrix metalloproteinase 23 gene (MMP23A and $-B$ ) to investigate further because this gene is involved in bone matrix resorption and bone remodeling. ${ }^{31}$ 


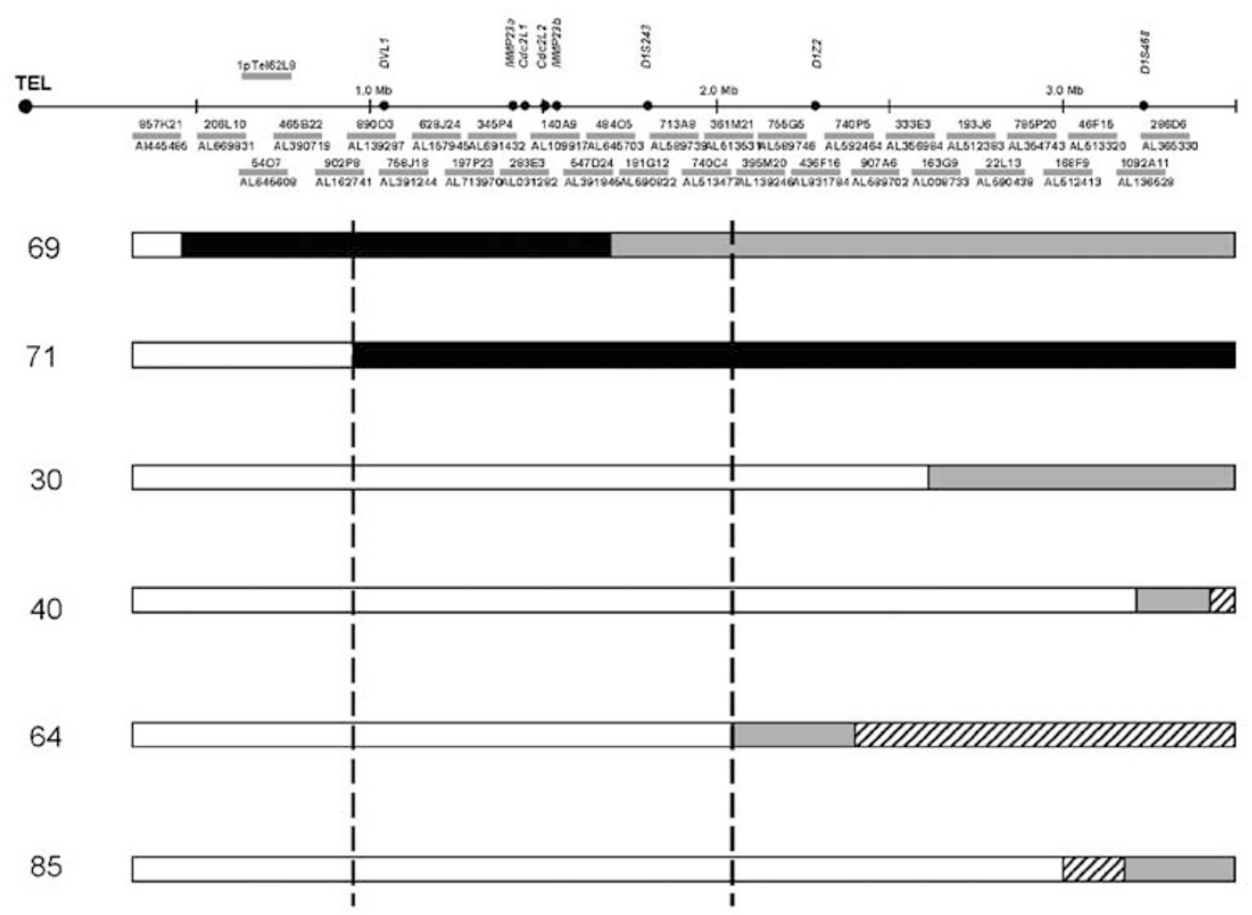

Figure 3 Alignment of the deletions, duplications, and triplications for six subjects as defined by array CGH and FISH. Distal 1 p36 is shown as a thin horizontal line. BAC clones from the distal 1 p36 contig are shown from telomeric (left) to centromeric (right). For each subject, white horizontal bars indicate deletion, black horizontal bars indicate triplication, gray horizontal bars indicate duplication, and hatched horizontal bars indicate the normal intact regions. The vertical dashed lines represent the distal (left) and proximal (right) boundaries of the critical region for craniosynostosis/large, late-closing anterior fontanel. The location of MMP23A and $-B$ is shown on the thin horizontal line representing distal 1 p36. MMP23A and $-B$ locate to the triplicated regions in subjects 69 and 71 with craniosynostosis and the deleted regions in subjects 30 and 40 with large, lateclosing anterior fontanels. Subjects 64 and 85 had unremarkable suture closure.

\section{In situ hybridization studies of $M m p 23$}

We studied the expression pattern of Mmp23 in mouse calvarial sutures during embryonic development. Our results demonstrate that $M m p 23$ was expressed in the osteoblasts lining the calvarial bones, in the osteogenic fronts of the bones that are sites of active osteogenesis, and expressed weakly in the suture mesenchyme (Figure 5).

\section{Discussion}

Chromosome rearrangements have played a critical role in syndrome delineation and gene identification. In order to utilize chromosome rearrangements for genetic/genome analysis, the rearrangements must be defined and deletion/ duplication boundaries delineated. Molecular cytogenetic methods have been instrumental in delineating rearrangements. Numerous examples have been published in which deletions, translocations, and other rearrangements have been used to identify disease-causing genes (eg, 1,3,50,51).

One recent technique used to identify chromosome imbalance is array CGH. Over 200 reports have been published using this technique (eg, 52-58). However, although regions of imbalance are revealed, the structural configuration of the abnormal chromosome is not. FISH can be used to establish the precise orders and orientations of the rearranged segments. This allows for possible mechanisms for causing each rearrangement to be proposed. In addition, by comparing the known structures of the rearrangements a critical region for candidate genes can be established.

\section{Mechanisms for generating complex rearrangements of chromosome 1}

Recently, we proposed a premeiotic model for the generation of complex rearrangements of $1 \mathrm{p} 36 .{ }^{19}$ Central to the mechanism is successive breakage-fusion-bridge (BFB) cycles. McClintock first proposed that in maize, broken chromosomes undergo a cycle of end-to-end fusions followed by random breakage of the newly created dicentric chromosome until the chromosome becomes stabilized through acquisition of a telomere. ${ }^{59,60}$ This telomere could be obtained through de novo synthesis of the telomeric repeat (TTAGGG) ${ }_{n}$ through telomerasedependent or independent mechanisms. ${ }^{19}$ In addition, telomeres could be acquired from another chromosome 


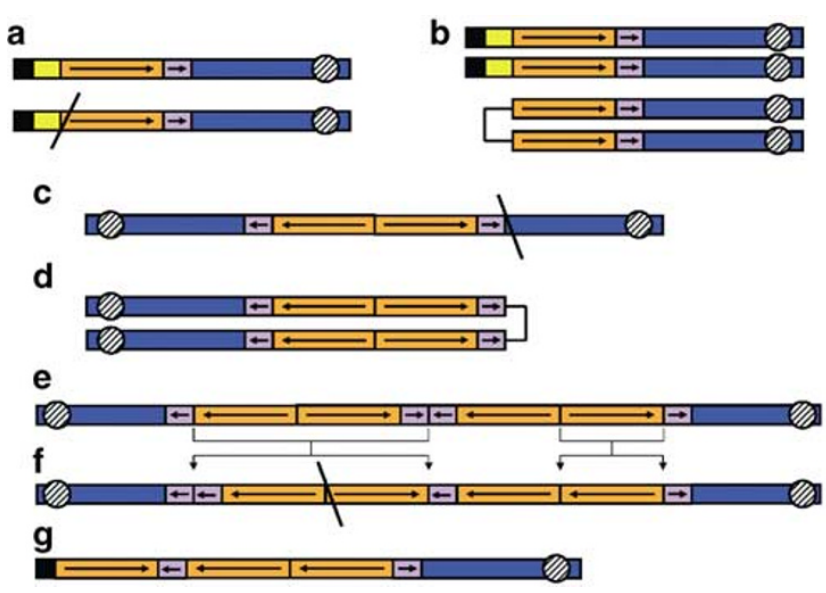

Figure 4 Possible mechanism for the complex rearrangement in subject 71. (a) Double-strand break in one chromosome 1 causes a terminal deletion. (b) After replication, the sister chromatids of the deleted chromosome 1 fuse and the resulting dicentric chromosome undergoes the first of two breakage-fusion-bridge (BFB) cycles. (c) During first anaphase, the newly formed dicentric chromosome breaks at a random location, indicated by a diagonal line. The resulting chromosome contains an inverted duplication. (d) After replication, the sister chromatids fuse. (e) The dicentric chromosome undergoes an inversion of each of the segments indicated by vertical arrows. (f) A second break occurs during cell division and the dicentric chromosome breaks at a random site, indicated by a diagonal line. (g) The broken, rearranged chromosome 1 acquires the subtelomeric region of $19 q$ through a telomere capture event (type of event not specified).

end by a variety of mechanisms. ${ }^{61}$ We propose that in subject 69, the deleted chromosome 1 underwent three BFB cycles and three inversions before becoming structurally stable (data not shown). Similarly, subject 71 underwent two BFB cycles and two inversions before a telomere was acquired from 19q (Figure 4). Although several mechanisms exist for acquiring a nonhomologous telomere (reviewed in Ballif $e a^{37}$ ), in subject 71, we propose a break-induced replication mechanism for telomere acquisition. In subject 64 , as well as in previously published subjects 30 and $40,{ }^{19}$ one BFB cycle was sufficient to derive the abnormal chromosomes 1 , whereas the rearrangement in subject 85 required two BFB cycles and two inversions to derive the final aberrant structure (data not shown). The various steps required are predicted on the idea of Occam's razor; they represent the simplest sequence of events that could generate the rearrangements in each subject. ${ }^{62,63}$

\section{MMP23 plays a role in regulation of cranial suture closure}

For many dosage-sensitive genes, mutations may lead to an isolated clinical phenotype, while in contiguous gene deletion syndromes (CGS), chromosome imbalance may alter the expression of several dosage-sensitive genes. ${ }^{8}$ As presented here, the characterization of complex rearrangements of $1 \mathrm{p}$ has identified a region likely to contain a gene involved in cranial suture closure regulation.

The calvarial bones, which form the upper vault of the human skull, are connected by thin fibrous tissue called sutures. During human postnatal calvarial development, the sutures fuse in a synchronized manner, regulated by a complex system of bone formation and resorption. The premature fusion of these joints is called craniosynostosis. ${ }^{64,65}$ Craniosynostosis is one of the most common human malformations, occurring in approximately one in 2500-3000 (0.03-0.04\%) newborns. ${ }^{66}$ Mutations in several genes have been identified as causes for craniosynostosis. A mutation in MSX2 was discovered in a family with autosomal dominant craniosynostosis, ${ }^{67}$ and the TWIST and fibroblast growth factor receptors $1,-2$, and -3 (FGFR1, -2, -3) have also been implicated in various syndromes involving craniosynostosis. ${ }^{64}$

The matrix metalloproteinase-23 (MMP23) genes, $A$ and $B$, belong to a large family of genes involved in extracellular matrix (ECM) remodeling. These metalloproteinase genes, of which there are four distinct families - collagenases, gelatinases, stromelysins, and membrane-type MMPs (MT-MMPs) - also play a function in connective tissue remodeling in bone growth and embryonic development. ${ }^{68} M M P 23 A$ and its duplicated copy $-B$ are located $\sim 0.5-1.0 \mathrm{~kb}$ from the $3^{\prime}$ end of the $C d c 2 L 1-2$ locus, which occupies $\sim 120 \mathrm{~kb}$ on $1 \mathrm{p} 36.3 .^{69}$ The two copies of MMP23 share $\sim 90 \%$ sequence identity. Although MMP23 belongs to the metalloproteinase gene family, the gene does not fit into any of the subfamilies, instead being classified among several MMPs in the 'other MMPs' group. ${ }^{70}$ Although it contains a number of protein domains that are typical of MMPs - including a prodomain, catalytic domain, hinge region, and a C-terminal domain - MMP23A and $-B$ are missing a signal sequence at the N-terminus which is characteristic of other MMPs. ${ }^{70}$ In addition, each gene's prodomain is significantly shorter than most MMPs, although it does contain the cysteine residue necessary for ligation of zinc ions and is essential for maintaining latency. ${ }^{70}$ The residues connecting the prodomains and catalytic domains further suggest an activating mechanism different from most MMPs, which has been shown in MTMMPs and stromelysin-3. ${ }^{70} M M P 23 A$ and $-B$ are expressed in numerous tissues, including placenta, ovary, testis, prostate, heart, and pancreas. ${ }^{69,70}$ In addition, using gene expression analysis of hBMP2 injected in vivo into mouse quadriceps to induce bone formation, Clancy et $\mathrm{al}^{31}$ showed increased expression of Mmp23 in osteoblasts and chrondrocytes, suggesting a role for $M m p 23$ in bone formation and remodeling.

We found that Mmp23 is expressed during calvarial bone development in mouse. The Mmp23 transcripts localized 


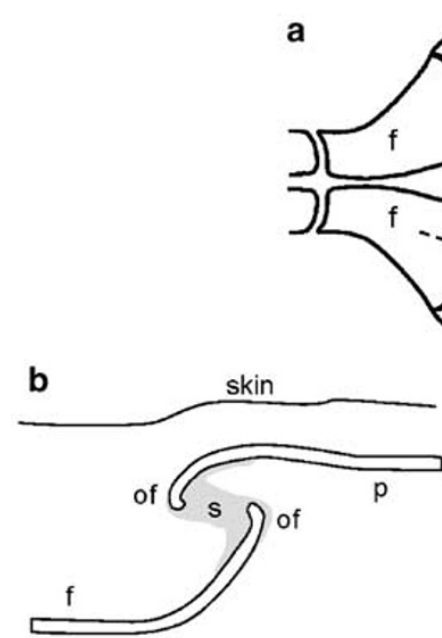

d

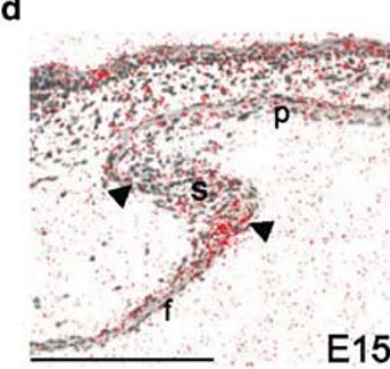

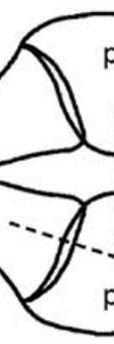

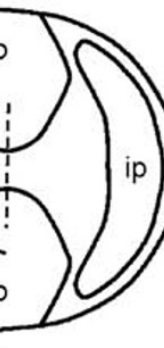

C

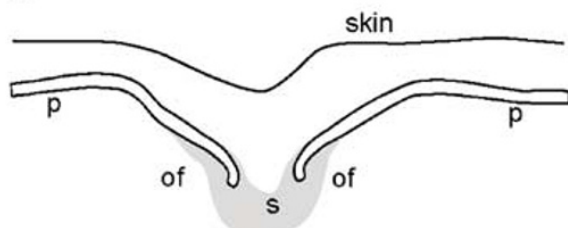

e

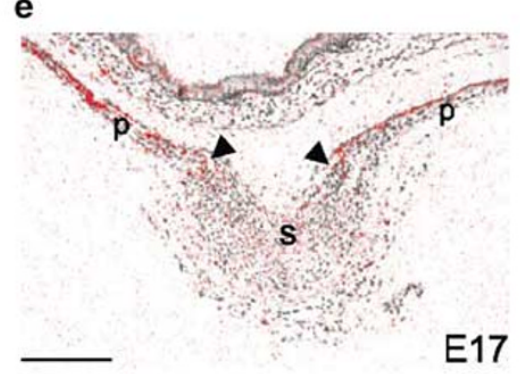

Figure 5 In situ hybridization of Mmp23 to mouse calvarial sutures. (a) Schematic diagram of the apical view of a mouse embryonic day 17 calvaria. Dotted lines indicate the plane of section made for histological sections of coronal and sagittal sutures. (b, c) Schematic diagrams illustrating the coronal (b) and sagittal (c) sutures. Note that in the coronal suture the bone ends of the parietal and frontal bones overlap. (d, e) Both the coronal (d) and sagittal (e) sutures show similar patterns of expression. Mmp23 is expressed in the osteoblasts lining the frontal and parietal bones, at the osteogenic fronts of these bones (arrowheads), and to a lesser extent in the suture mesenchyme. (f) frontal bone, (ip) interparietal bone, (of) osteogenic fronts, (p) parietal bone, (s) suture mesenchyme. Scale bars $200 \mu \mathrm{m}$.

not just to mature osteoblasts lining the calvarial bones but also to the preosteoblasts in the osteogenic fronts, and to a lesser extent the suture mesenchyme (Figure 5). We hypothesize that overexpression of MMP23 through increased gene copy number results in craniosynostosis, whereas decreased expression of MMP23 through deletion of one copy results in large, late-closing fontanels. Because cranial suture formation relies on a complex pathway of bone modeling and resorption events involving a large number of genes, we cannot conclude whether MMP23 plays a primary role in cranial suture patency and closure or is one of several genes which plays a secondary role. Nonetheless, the mouse calvarial bone expression pattern supports a role for MMP23 in the regulation of suture fate.

\section{Conclusion}

The most complex and/or rare cytogenetic rearrangements are often crucial for the identification of clinically relevant dosage-sensitive genes. These rearrangements may allow for narrowing of critical regions for identifying candidate genes and, in the most singular of cases, disruption of disease-causing genes. This allows for greater understanding of gene function in causing clinical conditions and may lead to therapeutic strategies for affected individuals. Our work further demonstrates the usefulness of cytogenetic aberrations for gene identification. We have used complex rearrangements of 1 p36 to further our understanding of the mechanisms resulting in terminal rearrangements and to identify a candidate gene for regulation of cranial suture closure.

\section{Electronic database information}

Ensembl: http://www.ensembl.org

NCBI genome browser: http://www.ncbi.nlm.org

UCSC genome browser: http://genome.ucsc.edu

\section{Acknowledgements}

We thank the subjects and their families for their participation in this research. We thank Dr Helen E Roberts, Norfolk, VA, for clinical information on subject 64. We also thank Dr J Ohnishi (Hokkaido 
University, Sapporo, Japan) for the Mmp23 mRNA probe. This study was supported in part by grants from the NIH National Institute of Deafness and Other Communication Disorders K08 DC00169 (HAH) and NIH National Institute for Child Health and Development PO1 HD39420 (LGS).

\section{References}

1 Francke U: Regional localization of the human genes for malate dehydrogenase-1 and isocitrate dehydrogenase-1 on chromosome 2 by interspecific hybridization using human cells with the balanced reciprocal translocation $\mathrm{t}(1 ; 2)$ (q32; $\mathrm{q} 13$ ). Cytogenet Cell Genet 1975; 14: 308-312.

2 Nickerson E, Greenberg F, Keating MT, McCaskill C, Shaffer LG: Deletions of the elastin gene at 7q11.23 occur in approximately 90\% of patients with Williams syndrome. Am J Hum Genet 1995; 56: $1156-1165$.

3 Ewart AK, Morris CA, Atkinson D et al: Hemizygosity at the elastin locus in a developmental disorder, Williams syndrome. Nat Genet 1993; 5: 11-16.

4 Breuning MH, Dauwerse HG, Fugazza G et al: Rubinstein-Taybi syndrome caused by submicroscopic deletions within $16 \mathrm{p} 13.3$. Am J Hum Genet 1993; 52: 249-254.

5 Ballabio A, Andria G: Deletions and translocations involving the distal short arm of the human $\mathrm{X}$ chromosome: review and hypotheses. Hum Mol Genet 1992; 1: 221-227.

6 Stankiewicz P, Lupski JR: Genome architecture, rearrangements and genomic disorders. Trends Genet 2002; 18: 74-82.

$7 \mathrm{Wu}$ YQ, Sutton VR, Nickerson E et al: Delineation of the common critical region in Williams syndrome and clinical correlation of growth, heart defects, ethnicity, and parental origin. Am J Med Genet 1998; 78: 82-89.

8 Shaffer LG, Lupski JR: Molecular mechanisms for constitutional chromosomal rearrangements in humans. Annu Rev Genet 2000; 34: $297-329$.

9 Heilstedt HA, Ballif BC, Howard LA, Kashork CD, Shaffer LG: Population data suggest that deletions of $1 \mathrm{p} 36$ are a relatively common chromosome abnormality. Clin Genet 2003; 64: $310-316$.

10 Shapira SK, McCaskill C, Northrup H et al: Chromosome 1p36 deletions: the clinical phenotype and molecular characterization of a common newly delineated syndrome. Am J Hum Genet 1997; 61: $642-650$.

11 Slavotinek A, Shaffer LG, Shapira SK: Monosomy 1p36. J Med Genet 1999; 36: 657-663.

12 Heilstedt HA, Ballif BC, Howard LA et al: Physical map of 1p36, placement of breakpoints in monosomy 1p36, and clinical characterization of the syndrome. Am J Hum Genet 2003; 72: $1200-1212$.

$13 \mathrm{Wu}$ YQ, Heilstedt HA, Bedell JA et al: Molecular refinement of the 1 p36 deletion syndrome reveals size diversity and a preponderance of maternally derived deletions. Hum Mol Genet 1999; 8: 313-321.

14 Colmenares C, Heilstedt HA, Shaffer LG et al: Loss of the SKI proto-oncogene in individuals affected with 1 p36 deletion syndrome is predicted by strain-dependent defects in Ski-/mice. Nat Genet 2002; 30: 106-109.

15 Heilstedt HA, Burgess DL, Anderson $\mathrm{AE}$ et al: Loss of the potassium channel beta-subunit gene, KCNAB2, is associated with epilepsy in patients with $1 \mathrm{p} 36$ deletion syndrome. Epilepsia 2001; 42: 1103-1111.

16 Heilstedt HA, Shapira SK, Gregg AR, Shaffer LG: Molecular and clinical characterization of a patient with duplication of 1 p36.3 and metopic synostosis. Clin Genet 1999; 56: 123-128.

17 Garcia-Heras J, Corley N, Garcia MF, Kukolich MK, Smith KG, Day DW: De novo partial duplications 1p: report of two new cases and review. Am J Med Genet 1999; 82: 261-264.

18 Warden CR, Pillers DA, Rice MJ et al: Interstitial duplication of the short arm of chromosome 1 in a newborn with congenital heart disease and multiple malformations. Am J Med Genet 2001; 101: $100-105$.

19 Ballif BC, Yu W, Shaw CA, Kashork CD, Shaffer LG: Monosomy 1p36 breakpoints suggest pre-meiotic breakage-fusion-bridge cycles are involved in generating terminal deletions. Hum Mol Genet 2003; 12: 2153-2165.

$20 \mathrm{Yu} \mathrm{W}$, Ballif BC, Kashork CD, Heilstedt HA, Shaw CA, Shaffer LG: Development of a comparative genomic hybridization microarray and demonstration of its utility with 25 wellcharacterized human 1p36 deletions. Hum Mol Genet 2003; 12: $2145-2152$.

21 Wessendorf S, Fritz B, Wrobel G et al: Automated screening for genomic imbalances using matrix-based comparative genomic hybridization. Lab Invest 2002; 82: 47-60.

22 Ballif BC, Kashork CD, Shaffer LG: FISHing for mechanisms of cytogenetically defined terminal deletions using chromosome-specific subtelomeric probes. Eur J Hum Genet 2000; 8: $764-770$.

23 Shaffer LG, McCaskill C, Han JY et al: Molecular characterization of de novo secondary trisomy 13. Am J Hum Genet 1994; 55: 968-974.

$24 \mathrm{O}^{\prime}$ Brien TW: Evolution of a protein-rich mitochondrial ribosome: implications for human genetic disease. Gene 2002; 286: $73-79$.

25 Fitzgerald J, Tay Ting S, Bateman JF: WARP is a new member of the von Willebrand factor A-domain superfamily of extracellular matrix proteins. FEBS Lett 2002; 517: 61-66.

26 Fitzgerald J, Bateman JF: Is there an evolutionary relationship between WARP (von Willebrand factor A-domain-related protein) and the FACIT and FACIT-like collagens? FEBS Lett 2003; 552: 91-94.

27 Ogura T, Wilkinson AJ: AAA+ superfamily ATPases: common structure - diverse function. Genes Cells 2001; 6: 575-597.

28 Yoshida Y, Tanaka S, Umemori $\mathrm{H}$ et al: Negative regulation of BMP/Smad signaling by Tob in osteoblasts. Cell 2000; 103: 1085-1097.

29 Yoshida Y, von Bubnoff A, Ikematsu N et al: Tob proteins enhance inhibitory Smad-receptor interactions to repress BMP signaling. Mech Dev 2003; 120: 629-637.

30 Visse R, Nagase H: Matrix metalloproteinases and tissue inhibitors of metalloproteinases: structure, function, and biochemistry. Circ Res 2003; 92: 827-839.

31 Clancy BM, Johnson JD, Lambert A-J et al: A gene expression profile for endochondral bone formation: oligonucleotide microarrays establish novel connections between known genes and BMP-2-induced bone formation in mouse quadriceps. Bone 2003; 33: 46-63.

32 Lahti JM, Xiang J, Kidd VJ: The PITSLRE protein kinase family. Prog Cell Cycle Res 1995; 1: 329-338.

33 Lahti JM, Valentine M, Xiang J et al: Alterations in the PITSLRE protein kinase gene complex on chromosome 1p36 in childhood neuroblastoma. Nat Genet 1994; 7: 370-375.

34 Ishida N, Kawakita M: Molecular physiology and pathology of the nucleotide sugar transporter family (SLC35). Pflugers Arch 2003; 447: $768-775$.

35 Lerner F, Niere M, Ludwig A, Ziegler M: Structural and functional characterization of human NAD kinase. Biochem Biophys Res Commun 2001; 288: 69-74.

36 Cabrera-Vera TM, Vanhauwe J, Thomas TO et al: Insights into G protein structure, function, and regulation. Endocr Rev 2003; 24: $765-781$.

37 Downes GB, Gautam N: The G protein subunit gene families. Genomics 1999; 62: 544-552.

38 Mihalek RM, Banerjee PK, Korpi ER et al: Attenuated sensitivity to neuroactive steroids in gamma-aminobutyrate type A receptor delta subunit knockout mice. Proc Natl Acad Sci USA 1999; 96: $12905-12910$.

39 Leitges M, Sanz L, Martin P et al: Targeted disruption of the zetaPKC gene results in the impairment of the NF-kappaB pathway. Mol Cell 2001; 8: 771-780. 
40 Kaufman CD, Martinez-Rodriguez G, Hackett Jr PB: Ectopic expression of c-ski disrupts gastrulation and neural patterning in zebrafish. Mech Dev 2000; 95: 147-162.

41 Sato K, Sato M, Nakano A: Rer1p, a retrieval receptor for ER membrane proteins, recognizes transmembrane domains in multiple modes. Mol Biol Cell 2003; 14: 3605-3616.

42 Warren DS, Morrell JC, Moser HW, Valle D, Gould SJ: Identification of PEX10, the gene defective in complementation group 7 of the peroxisome-biogenesis disorders. Am J Hum Genet 1998; 63: 347-359.

43 Zhou B, Westaway SK, Levinson B, Johnson MA, Gitschier J, Hayflick SJ: A novel pantothenate kinase gene (PANK2) is defective in Hallervorden-Spatz syndrome. Nat Genet 2001; 28: $345-349$.

44 Iso T, Kedes L, Hamamori Y: HES and HERP families: multiple effectors of the Notch signaling pathway. J Cell Physiol 2003; 194: $237-255$.

45 Ross SE, Greenberg ME, Stiles CD: Basic helix-loop-helix factors in cortical development. Neuron 2003; 39: 13-25.

46 Zine A: Molecular mechanisms that regulate auditory hair-cell differentiation in the mammalian cochlea. Mol Neurobiol 2003; 27: $223-238$.

47 Montgomery RI, Warner MS, Lum BJ, Spear PG: Herpes simplex virus- 1 entry into cells mediated by a novel member of the TNF/ NGF receptor family. Cell 1996; 87: 427-436.

48 Marsters SA, Ayres TM, Skubatch M, Gray CL, Rothe M, Ashkenazi A: Herpesvirus entry mediator, a member of the tumor necrosis factor receptor (TNFR) family, interacts with members of the TNFR-associated factor family and activates the transcription factors NF-kappaB and AP-1. J Biol Chem 1997; 272: 14029-14032.

49 Vainio S, Jalkanen M, Vaahtokari A et al: Expression of syndecan gene is induced early, is transient, and correlates with changes in mesenchymal cell proliferation during tooth organogenesis. Dev Biol 1991; 147: 322-333.

50 Francke U, Ochs HD, de Martinville B et al: Minor Xp21 chromosome deletion in a male associated with expression of Duchenne muscular dystrophy, chronic granulomatous disease, retinitis pigmentosa and McLeod syndrome. Am J Hum Genet 1985; 37: 250-267.

51 Wu YQ Badano JL, McCaskill C, Vogel H, Potocki L, Shaffer LG: Haploinsufficiency of ALX4 as a potential cause of parietal foramina in the $11 \mathrm{p} 11.2$ contiguous gene-deletion syndrome. Am J Hum Genet 2000; 67: 1327-1332.

52 Solinas-Toldo S, Lampel S, Stilgenbauer S et al: Matrix-based comparative genomic hybridization: biochips to screen for genomic imbalances. Genes Chromosomes Cancer 1997; 20: 399-407.

53 Pinkel D, Segraves R, Sudar D et al: High resolution analysis of DNA copy number variation using comparative genomic hybridization to microarrays. Nat Genet 1998; 20: 207-211.

54 Albertson DG, Ylstra B, Segraves R et al: Quantitative mapping of amplicon structure by array CGH identifies CYP24 as a candidate oncogene. Nat Genet 2000; 25: 144-146.
55 Buckley PG, Mantripragada KK, Benetkiewicz $\mathrm{M}$ et al: A fullcoverage, high-resolution human chromosome 22 genomic microarray for clinical and research applications. Hum Mol Genet 2002; 11: 3221-3229.

56 Veltman JA, Schoenmakers EF, Eussen BH et al: High-throughput analysis of subtelomeric chromosome rearrangements by use of array-based comparative genomic hybridization. Am J Hum Genet 2002; 70: 1269-1276.

57 Albertson DG: Profiling breast cancer by array CGH. Breast Cancer Res Treat 2003; 78: 289-298.

58 Paris PL, Albertson DG, Alers JC et al: High-resolution analysis of paraffin-embedded and formalin-fixed prostate tumors using comparative genomic hybridization to genomic microarrays. Am I Pathol 2003; 162: $763-770$.

59 McClintock B: The behavior of successive nuclear divisions of a chromosome broken at meiosis. Proc Natl Acad Sci USA 1939; 25: $405-416$.

60 McClintock B: The stability of broken ends of chromosomes in Zea mays. Genetics 1941; 26: 234-282.

61 Ballif BC, Wakui K, Gajecka M, Shaffer LG: Translocation breakpoint mapping and sequence analysis in three monosomy $1 \mathrm{p} 36$ subjects with der $(1) \mathrm{t}(1 ; 1)(\mathrm{p} 36 ; \mathrm{q} 44)$ suggest mechanisms for telomere capture in stabilizing de novo terminal rearrangements. Hum Genet 2004; 114: 198-206.

62 Thorburn WM: Occam's razor. Mind 1915; 24: 287-288.

63 Astbury C, Eichler E, Christ L, Schwartz S: Delineation of deletions and complexity in 'balanced' chromosome rearrangements: Occam's Razor bites the dust. Am J Hum Genet 2003; 73A: 203, (abstract).

64 Rice D, Rice R, Thesleff I: Molecular mechanisms in calvarial bone and suture development, and their relation to craniosynostosis. Eur J Orthod 2003; 25: 139-148.

65 Cohen Jr MM: Malformations of the craniofacial region: evolutionary, embryonic, genetic, and clinical perspectives. Am $J$ Med Genet 2002; 115: 245-268.

66 Cohen Jr MM, MacLean R (eds) Craniosynostosis: diagnosis, evaluation, and management. Oxford: Oxford University Press, 2002.

67 Jabs EW, Muller U, Li X et al: A mutation in the homeodomain of the human MSX2 gene in a family affected with autosomal dominant craniosynostosis. Cell 1993; 75: 443-450.

68 Birkedal-Hansen H, Moore WG, Bodden MK et al: Matrix metalloproteinases: a review. Crit Rev Oral Biol Med 1993; 4: 197-250.

69 Gururajan R, Grenet J, Lahti JM, Kidd VJ: Isolation and characterization of two novel metalloproteinase genes linked to the $C d c 2 L$ locus on human chromosome 1p36.3. Genomics 1998; 52: $101-106$.

70 Velasco G, Pendás AM, Fueyo A, Knäupert V, Murphy G, LópezOtín C: Cloning and characterization of human MMP-23, a new matrix metalloproteinase predominantly expressed in reproductive tissues and lacking conserved domains in other family members. J Biol Chem 1999; 274: 4570-4576. 